\title{
A Computationally Feasible DFT/CCSD(T) Correction Scheme for the Description of Weakly Interacting Systems
}

\author{
Miroslav Rubeša ${ }^{\mathrm{a}}$, Pavel Soldán ${ }^{\mathrm{b}}$, Petr Nachtigall ${ }^{\mathrm{a}}$ and Ota Bludský ${ }^{* a}$ \\ anstitute of Organic Chemistry and Biochemistry, Academy of Sciences of the Czech Republic and Center for \\ Biomolecules and Complex Molecular Systems, Flemingovo n. 2, CZ-16610 Prague, Czech Republic \\ ${ }^{\mathrm{b}}$ Charles University in Prague, Faculty of Mathematics and Physics, Department of Chemical Physics and Optics, \\ Ke Karlovu 3, 12116 Prague 2, Czech Republic
}

\begin{abstract}
A computationally feasible DFT/CCSD(T) correction scheme is proposed for precise calculations (close to the $\operatorname{CCSD}(\mathrm{T})$ accuracy) of weakly interacting molecular clusters. This approach formally falls within the DFTD class of methods (empirically corrected DFT methods), however, there are several important differences between the $\mathrm{DFT} / \mathrm{CCSD}(\mathrm{T})$ scheme proposed here and a standard DFTD approach: (i) it is parameter free, (ii) it does not use any damping functions, and (iii) the error of DFT is assumed to be anisotropic in general. In addition, the proposed $\mathrm{DFT} / \mathrm{CCSD}(\mathrm{T})$ correction scheme allows the analysis of assumptions commonly used in the DFTD calculations. Application of this method on the ethylene-benzene and benzene-benzene complexes leads to the conclusion that interaction energies obtained with the DFT/CCSD(T) correction scheme can be obtained with a near reference level accuracy with an error not exceeding $0.1 \mathrm{kcal} / \mathrm{mol}$. A proper choice of a reference set is shown to be more important than the anisotropy of the $\mathrm{DFT} / \mathrm{CCSD}(\mathrm{T})$ correction.
\end{abstract}

\section{INTRODUCTION}

One of the biggest drawbacks of the current density functional theory (DFT) is its inability to account for the dispersion interactions. It is, therefore, understandable that there is an ongoing research aimed at the inclusion of the dispersion interaction into DFT. The work in this direction can be divided into several classes: (i) finding a truly non-local exchange-correlation functional that can account for the dispersion interaction in a physically understandable manner (e.g., the doubly local density approximation or the Kohn's scheme, (Refs. [1-5]) (ii) intermolecular potentials based on the symmetry-adapted perturbation theory with dispersion energies from the time-dependent DFT [6-8] and (iii) describing the dispersion as the additional $R^{-6}$ term in an empirical way similar to that commonly used in the force-field calculations (these methods are commonly denoted as "DFTD” or “DFT-D", e.g., Refs. [9-14]).

A large progress has been achieved recently in all classes outlined above; each of these approaches has numerous advantages but also some disadvantages. The methods from the first two classes mostly suffer from relatively large computational demands, and their applications on the large molecular systems were not achieved yet. On the contrary, the methods from the third class (the DFTD methods) are computationally simple, however, the underlying physics is not very clear; that is connected to some drawbacks discussed below.

*Address correspondence to this author at the Institute of Organic Chemistry and Biochemistry, Academy of Sciences of the Czech Republic and Center for Biomolecules and Complex Molecular Systems, Flemingovo n. 2, CZ-16610 Prague, Czech Republic; Tel: +420-220410324; Fax: +420220410320; E-mail: ota.bludsky@uochb.cas.cz
Several DFTD schemes have been recently defined (see, e.g., Refs. [10-14]). The inter-molecular interaction energy is always calculated as

$$
E_{D F T D}=E_{D F T}+\Delta E
$$

where $\Delta E$ is an inter-molecular energy correction (usually, $\Delta E$ is denoted as $E_{\mathrm{disp}}$ ). All these schemes assume a pair-wise representability of the inter-molecular energy corrections:

$$
\Delta E=\sum_{i=1}^{N_{a}} \sum_{j=1}^{N_{b}} \Delta E_{i j},
$$

where $N_{a}$ and $N_{b}$ are numbers of atoms in the corresponding subsystems. Following the original strategy of Ahlrichs at el. [15] the correction term $\Delta E_{i j}$, represented by a leading term of the dispersion interaction, is used together with an artificial damping function in order to switch off the dispersion interaction at shorter nuclear separations:

$$
\Delta E_{i j}=-\frac{C_{i j}}{R_{i j}^{6}} f_{\text {damp }}\left(R_{i j}\right)
$$

Here, $C_{i j}$ and $R_{i j}$ are the dispersion coefficients and distances for atoms $i$ and $j$, and $f_{\text {damp }}$ stands for the damping function. Various forms of the damping function were used (for details see, e.g., Ref. [16]). While the use of the damping function is believed to be essential for the DFTD model, its particular form cannot be physically justified; clearly, the performance of the model depends on the choice of the damping function, as it has been shown (e.g., Ref. [11]). In addition to the difficulties with the choice of the damping function form, the inter-atomic coefficients $C_{i j}$ of the $R^{-6}$ term must be derived. Several strategies, mostly relying on 
some combination rules, were suggested and used within the DFTD framework, assuming isotropic atomic polarizabilities.

As it has been pointed out previously [10], $\Delta E$ is just a model-dependent quantity and it has no physical meaning. Therefore, $\Delta E$ depends on the exchange-correlation functional and on the basis set used in the model. Success or failure of the DFTD model depends on the fit of the $C_{i j}$ coefficients and damping function parameters in Equation (3). In addition, the reliability of the DFTD correction schemes depends on the validity of assumption of the isotropy of atomic polarizabilities. Clearly, within the DFTD scheme, the $\Delta E$ term does not account only for the DFT inability to describe any dispersion interaction; instead, the $R^{-6}$ term together with the damping function is a correction of the DFT method for the description of weakly interacting systems. The choice of the particular functional form of Eq. (3) may, at least in principle, simplify the fitting procedure, however, it introduces an artificial constraint on the parameter space. The reliability of the DFTD schemes depends on the fitting procedure used, including the definition of the training set.

It is apparent that the definition of $\Delta E$ in Eq. (1) can be generalized: $\Delta E$ includes all the DFT errors in the description of the weakly interacting systems. The reference system, for which precise calculations (e.g., $\operatorname{CCSD}(\mathrm{T})$ with a sufficiently flexible basis set) are feasible, can be used for the definition of $\triangle E$, and this correction can be applied to improve the DFT description of the large systems that can only be described at the DFT level. In this contribution we propose a computationally feasible DFT/CCSD(T) correction scheme that can be used for precise calculations (close to the $\operatorname{CCSD}(\mathrm{T})$ accuracy) of weakly interacting large molecules. This approach formally falls within the DFTD class of methods (the empirically corrected DFT methods), however, there are several important differences between the DFT/CCSD(T) scheme proposed here and general DFTD approach. (i) The artificial assumption about the particular form of Eq. (3) is lifted, instead, all the deficiencies of the DFT method for the description of weakly interacting systems are controlled purely numerically (using an interpolation method), by correcting the DFT to the CCSD(T) accuracy. (ii) The deficiencies of DFT are assumed to be anisotropic in general, (iii) the $\mathrm{DFT} / \mathrm{CCSD}(\mathrm{T})$ correction scheme is parameter free, its accuracy depends only on the choice of a reference system (equivalent of a training set in DFTD).

In this paper the importance of these generalizations is investigated and discussed. It should be stressed that the $\mathrm{DFT} / \mathrm{CCSD}(\mathrm{T})$ correction scheme should not be viewed as the dispersion correction. The dispersion energy together with the electrostatic interaction certainly dominates the DFT correction for the larger inter-system separation, however, when electron densities of the interacting systems start to overlap, there are other sources of error than the dispersion effecting the DFT performance. DFT/CCSD(T) correction scheme represents a suitable framework for analysis of the DFT error, and it can help in further development and improvement of the DFTD approaches. The validity of assumptions adopted in the currently implemented DFTD schemes, such as the parameter transferability or isotropy of $\Delta E$, are analyzed using the proposed correction scheme. Last but not least, it is demonstrated that the DFT/CCSD(T) scheme is capable to reproduce the results of the $\operatorname{CCSD}(\mathrm{T})$ calculations for relevant parts of the potential energy surface (PES) with the DFT computational demands.

\section{METHODS}

\section{$\Omega$-DFTD Method}

Quite generally, the correction function $\Delta E=E_{C C S D(T)}-E_{D F T}$ can be expressed in terms of inter-molecular coordinates such as the monomer-monomer distance $R$ and angular parameters $\Omega$ describing their mutual orientation. In the standard DFTD scheme (Refs. [10-14]) the correction is sought as a pair-wise approximation to $\Delta E$ replacing the $R, \Omega$ dependence by sum over the atom-atom distances (see Eq. 2 ). This approximation was found reasonably accurate for the determination of the equilibrium structures and interaction energies of most weakly bound molecular complexes. Nevertheless, failure of this approach becomes inevitable in the repulsion region where angular dependences start to reflect the local symmetry of the overlapping electronic densities of interacting molecules. Thus, in our formulation of the DFTD method we keep the $\Omega$-dependence of the atomic pair-wise corrections

$$
\Delta E(R, \Omega)=\sum_{i}^{N_{a}} \sum_{j}^{N_{b}} \varepsilon_{i j}^{\Omega}\left(r_{i j}\right),
$$

where $\varepsilon_{i j}^{\Omega}$ are the $\Omega$-dependent pair-wise correction functions and $r_{i j}$ is the distance between atoms $i$ and $j$ belonging to different monomers. Similar to the "standard" onedimensional approaches we assume $R^{-6}+R^{-8}$ asymptotic behavior of the $\Omega$-dependent correction surfaces. The parameters of correction functions in the DFTD scheme are usually obtained from high-level ab initio calculations (typically at the $\operatorname{CCSD}(\mathrm{T})$ level) by a least-squares fitting of the equilibrium geometries and energies of the carefully selected model structures (training set). Basically, the parameters of the $\varepsilon_{i j}^{\Omega}$ functions can be determined in similar way. In $\Omega$-dependent formulation, however, global potential energy surfaces (PES) of model systems are required, and the number of parameters would easily exceed manageable size. Here we propose a more general formulation of the DFTD scheme - an $\Omega$ dependent DFTD approach referred as the DFT/CCSD(T) or $\Omega$-DFTD computational scheme - defined in the following way: (i) the counterpoise corrected PES calculation of the model systems at the DFT and CCSD(T) levels, (ii) transforming correction surfaces $\Delta E$ from $R, \Omega$ to the $r_{i j}, \Omega_{i j}$ coordinate space using the Reciprocal Power Reproducing Kernel Hilbert Space (RP-RKHS) interpolation [17, 18], (iii) evaluating the $\Delta E$ for the real system using the correction functions obtained in the previous step. Note that in the last step we assume the inter-system transferability of the $\mathrm{DFT} / \mathrm{CCSD}(\mathrm{T})$ corrections for each atomic type. Actually, the validity of this assumption is essential for all the DFTD methods. 
To perform the $R, \Omega \rightarrow r_{i j}, \Omega_{i j}$ transformation one has to define the relationship between the global $(\Omega)$ and local $\left(\Omega_{i j}\right)$ sets of the angular parameters describing the monomermonomer and atom(i)-atom(j) orientations, respectively. The first step is the attachment of an atom-fixed frame to the atom. The alignment of the atom-fixed axes should respect the local environment of the atom and is different for each atomic type in molecule $(\mathrm{H}, \mathrm{C}(\mathrm{sp}), \mathrm{C}(\mathrm{sp} 2)$, etc.). This can be achieved by various means, e.g., considering attached bonds or by using the electron density around atoms. In the following we will define unambiguously the atom-fixed frame associated with each atom type. The proposed computational strategy for the hydrogen and carbon $\left(\mathrm{sp}^{2}\right)$ atoms is outlined below on example of the $\mathrm{H}_{2}$ and ethylene molecules.

\section{$\mathrm{H}_{2} \ldots \mathrm{H}_{2}$}

Let us discuss in detail the proposed correction scheme using the $\mathrm{H}_{2}$ dimer as an example. At a fixed monomermonomer geometry the correction energy is expressed in a pair-wise additive approximation

$$
\Delta E(R, \Omega)=\varepsilon_{13}^{\Omega}\left(r_{13}\right)+\varepsilon_{14}^{\Omega}\left(r_{14}\right)+\varepsilon_{23}^{\Omega}\left(r_{23}\right)+\varepsilon_{24}^{\Omega}\left(r_{24}\right),
$$

where $R$ is the distance between the centers of masses of the hydrogen molecules, $\Omega$ represents the set of the angular parameters $\alpha, \beta, \delta$ which describe the monomer-monomer orientation (see Fig. (1)), and $r_{i j}$ is the distance between hydrogen atoms $i$ and $j$. The alignment of the atom-fixed axes is chosen so that the $z$ axis coincides with the direction of the $\mathrm{H}-\mathrm{H}$ bond. The $\Omega_{i j}$ angular parameters can be approximated by $\Omega$ provided that $R$ is large with respect to the intramonomer $\mathrm{H}-\mathrm{H}$ distance (i.e., $\Delta E(R, \Omega)$ is a slowly varying function of $\Omega$ ). This approximation greatly simplifies the evaluation of the correction functions since they can be treated independently for each $\Omega$. Thus, Eq. 5 now reads

$\Delta E(R, \Omega)=\varepsilon_{H H}^{\Omega}\left(r_{13}\right)+\varepsilon_{H H}^{\Omega}\left(r_{14}\right)+\varepsilon_{H H}^{\Omega}\left(r_{23}\right)+\varepsilon_{H H}^{\Omega}\left(r_{24}\right)$,

where the contributions from the $i$-th and $j$-th hydrogen atoms $\varepsilon_{i j}^{\Omega}$ were replaced by an "averaged" correction function $\varepsilon_{H H}^{\Omega}$. The correction curve $\varepsilon_{H H}^{\Omega}$ is represented using the RPRKHS functional form

$\varepsilon_{H H}^{\Omega}(r)=\sum_{k} \alpha_{k}^{\Omega} q\left(r^{2}, r_{k}^{2}\right)$

with the kernel function

$q\left(r^{2}, r_{k}^{2}\right)=\frac{1}{3} \frac{1}{r_{>}^{6}}-\frac{1}{5} \frac{r_{<}^{2}}{r_{>}^{8}}$,

where $r$ is an the $\mathrm{H}-\mathrm{H}$ distance, $r_{k}$ are yet unspecified grid points in $r, r_{>}=\max \left(r, r_{k}\right), r_{<}=\min \left(r, r_{k}\right)$, and $\alpha_{k}^{\Omega}$ are interpolation coefficients. The interpolation coefficients $\alpha_{k}^{\Omega}$ are obtained as follows: for a fixed monomer-monomer orientation $\Omega$ we calculate the correction energies $\Delta E(R, \Omega)$ on a chosen grid in $R$; each grid point in $R$ generates four $\mathrm{H}-\mathrm{H}$ distances $r_{i j}$ thus inducing four grids in $r$; the grid points in $r$ are then substituted into the RP-RKHS functional form of the correction curve (Eqs. 7 and 8); finally, the four obtained functional forms of $\varepsilon_{H H}^{\Omega}$ together with the corresponding correction energies $\Delta E(R, \Omega)$ are substituted into the pair-wise additive approximation (Eq. 6). This leads to a system of linear equations for $\alpha_{k}^{\Omega}$, which is solved numerically using the Singular Value Decomposition (SVD) algorithm.

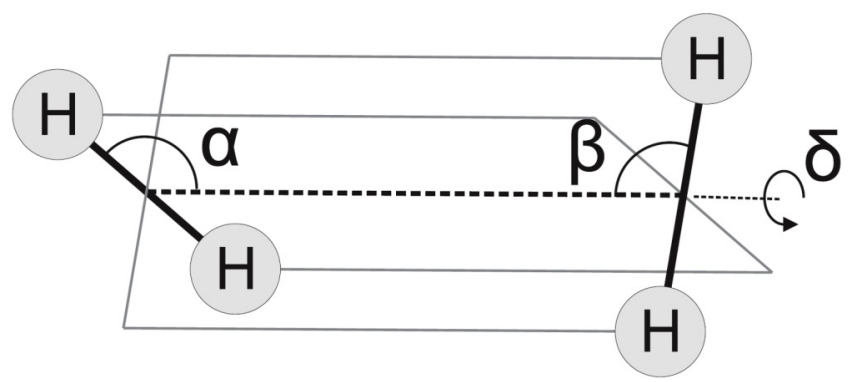

Fig. (1). Definition of the angular parameters $\alpha, \beta$, and $\delta$ describing the monomer-monomer orientation.

\section{$\mathrm{C}_{2} \mathrm{H}_{4} \ldots \mathrm{H}_{2}$}

Evaluation of the $\mathrm{C}\left(\mathrm{sp}^{2}\right)-\mathrm{H}$ correction curves proceeds in a very similar way as described for the $\mathrm{H}_{2} \ldots \mathrm{H}_{2}$ reference system. The total correction energy is expressed as a sum of the two terms corresponding to the $\mathrm{H}-\mathrm{H}$ and $\mathrm{C}-\mathrm{H}$ pair-wise interactions

$$
\Delta E(R, \Omega)=\Delta E_{C H}(R, \Omega)+\Delta E_{H H}(R, \Omega) .
$$

$\Delta E_{H H}$ is calculated using the correction surface obtained from the $\mathrm{H}_{2}-\mathrm{H}_{2}$ reference system (Eq. 6). The C-H contributions are evaluated as

$$
\begin{aligned}
& \Delta E_{C H}(R, \Omega)=\Delta E(R, \Omega)-\Delta E_{H H}(R, \Omega)= \\
& =\varepsilon_{C H}^{\Omega}\left(r_{13}\right)+\varepsilon_{C H}^{\Omega}\left(r_{14}\right)+\varepsilon_{C H}^{\Omega}\left(r_{23}\right)+\varepsilon_{C H}^{\Omega}\left(r_{24}\right),
\end{aligned}
$$

where $R$ is the distance between the centers of masses of the ethylene and hydrogen molecules, $r_{i j}$ is the distance between the carbon atom $i\left(\mathrm{C}_{2} \mathrm{H}_{4}\right)$ and hydrogen atom $j\left(\mathrm{H}_{2}\right)$, and $\Omega$ represents the set of the angular parameters $\alpha, \beta, \gamma, \delta$ describing the monomer-monomer orientation $(\alpha, \beta, \delta$ are defined in analogous way as in Fig. (1), $\gamma$ is angle of rotation of the ethylene molecule around the $\mathrm{C}-\mathrm{C}$ bond).

\section{$\mathrm{C}_{2} \mathrm{H}_{4} \ldots \mathrm{C}_{2} \mathrm{H}_{4}$}

The last step in this scheme is evaluation of the $\mathrm{C}\left(\mathrm{sp}^{2}\right)$ $\mathrm{C}\left(\mathrm{sp}^{2}\right)$ correction. The total correction energy is expressed as a sum of the three terms corresponding to the $\mathrm{H}-\mathrm{H}, \mathrm{C}-\mathrm{H}$, and C-C pair-wise interactions

$$
\Delta E(R, \Omega)=\Delta E_{C C}(R, \Omega)+\Delta E_{C H}(R, \Omega)+\Delta E_{H H}(R, \Omega) .
$$

$\Delta E_{H H}$ and $\Delta E_{C H}$ terms are calculated using the correction surfaces obtained from the $\mathrm{H}_{2} . . \mathrm{H}_{2}$ and $\mathrm{H}_{2} . . \mathrm{C}_{2} \mathrm{H}_{4}$ reference systems. The C-C contributions $\varepsilon_{C C}^{\Omega}\left(r_{i j}\right)$ are evaluated 
exactly in the same manner as in previous cases. $\Omega$-DFTD calculations employing the $\mathrm{H}_{2} \ldots \mathrm{H}_{2}, \mathrm{H}_{2} \ldots$ Eth, and Eth...Eth complexes in the reference set are denoted $\Omega^{(A)}$-DFTD.

\section{Isotropic $\Omega_{\mathrm{S}}$ Model}

In order to estimate an anisotropy of the DFT/CCSD(T) correction we employed the $\Omega$-fixed calculations using the reference set structures depicted in Fig. (2). These structures correspond to the "sandwich" (S) arrangement of the monomers that maximizes evaluated interactions (e.g., C-C for the sandwich structure of the ethylene dimer). The structures depicted in Fig. (2) are a natural choice for obtaining the one-dimensional correction curves using the RP-RKHS interpolation that we denote $\Omega_{S}$. Calculations of the onedimensional $\Omega_{S}$ corrections are straightforward since one only needs to evaluate one potential energy curve per a reference system. Provided that the atomic pair-wise corrections are isotropic ( $\Omega$ independent) these curves are exact counterparts of the DFTD corrections. Obviously, the $\Omega_{s}$ model preserves all advantages of the standard DFTD approach, but it is presumably much more robust since it does not involve any free parameters to adjust agreement with high level ab initio calculations.

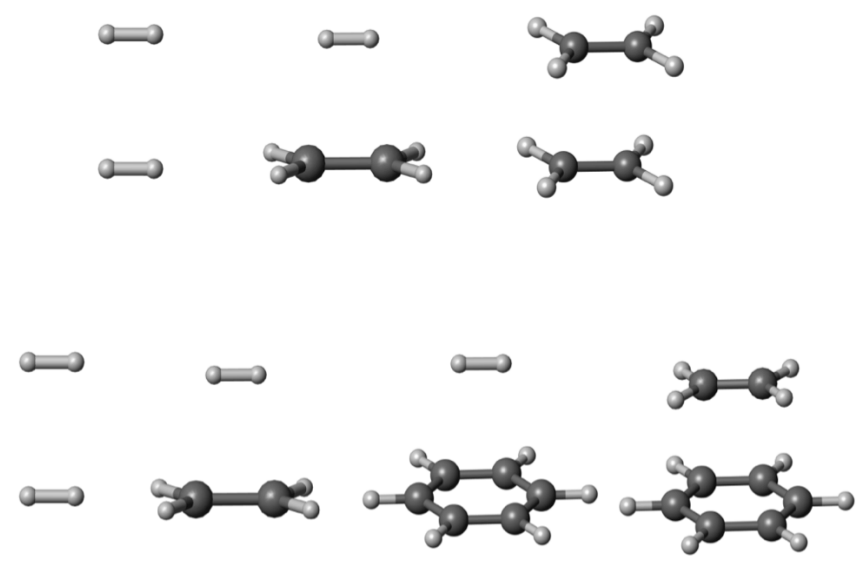

Fig. (2). Definition of the reference sets $\mathbf{A}$ (upper set) and $\mathbf{B}$ (lower set) used for the $\Omega_{S}^{(A)}$ - and $\Omega_{S}^{(B)}$-DFTD calculations, respectively.

Throughout this work we use two reference sets in $\Omega_{s}$ calculations: the reference set $\mathbf{A}$ consisting of the $\mathrm{H}_{2} \ldots \mathrm{H}_{2}$, $\mathrm{H}_{2} \ldots$ Eth, and Eth...Eth dimers used also for the anisotropic $\Omega^{(A)}$-DFTD calculations described in the previous section, and the reference set $\mathbf{B}$ consisting of the $\mathrm{H}_{2} \ldots \mathrm{H}_{2}, \mathrm{H}_{2} \ldots$ Eth, $\mathrm{H}_{2} \ldots \mathrm{Bz}$, and Eth...Bz dimers (Fig. 2); the corresponding results will be denoted as $\Omega_{S}^{(A)}$ and $\Omega_{S}^{(B)}$ for the reference sets A and $\mathbf{B}$, respectively. Comparison of the $\Omega_{S}$ calculations with reference sets $\mathbf{A}$ and $\mathbf{B}$ is valuable for providing insight into the nature of the DFT errors, namely the transferability of the DFT/CCSD(T) correction.

\section{Modeling Aromatic Systems}

The $\Omega$-DFTD method has been developed mainly for very large systems containing relatively small number of atomic types such as polycyclic aromatic hydrocarbons
(PAHs), graphite and carbon nanomaterials, etc. Generalization of the $\Omega$-DFTD method for complex systems containing many atomic types (like, e.g., DNA) is possible in principle, however, it would be highly impractical.

Aromatic hydrocarbons are among the most interesting and intensively studied benchmark systems. Unfortunately, there is no simple aromatic reference system for which high level $a b$ initio calculations of its global PES are currently feasible. Thus, the full $\Omega$ (anisotropic) calculations are performed only with the reference set A (see Fig. (2)).

Considering the reference set $\mathrm{A}$ as an example, the calculations for Eth...Bz proceed as follows. A sum of $24 \mathrm{H}-\mathrm{H}$ contributions $\Delta E_{H H}$ is calculated from the correction surface obtained from the $\mathrm{H}_{2} \ldots \mathrm{H}_{2}$ reference system. The angular parameters $\alpha, \beta, \delta$ are determined from the $\mathrm{H}-\mathrm{C}$ bond vectors for each $\mathrm{H}-\mathrm{H}$ term (the $z$ axis of atom-fixed frame coincides with the direction of the corresponding H-C bond). Similarly, $12 \mathrm{C}(\mathrm{Eth})-\mathrm{H}(\mathrm{Bz})$ and $24 \mathrm{C}(\mathrm{Bz})-\mathrm{H}(\mathrm{Eth})$ contributions obtained from the $\mathrm{H}_{2} \ldots$ Eth reference system give $\Delta E_{C H}$ using the $\alpha, \beta, \gamma, \delta$ angular parameters determined from the $\mathrm{H}-\mathrm{C}$ and $\mathrm{C}-\mathrm{C}$ bond vectors (the two $\mathrm{C}-\mathrm{C}$ bonds lying in the $\mathrm{C}\left(\mathrm{sp}^{2}\right)$ plane). Finally, a sum of $12 \mathrm{C}-\mathrm{C}$ contributions, $\Delta E_{C C}$, is obtained from the Eth...Eth reference system. The aromatic ring is modeled by ethylene molecules using one ethylene molecule per an aromatic $\mathrm{C}-\mathrm{C}$ bond. The interaction of two $\mathrm{C}\left(\mathrm{sp}^{2}\right)$ atoms is calculated from four different ethylene molecules oriented in the direction of the attached $\mathrm{C}-\mathrm{C}$ bonds in an aromatic system. The total correction is given by Eq. (11).

\section{Computational Details}

The CCSD(T) [19] calculations were carried out with the augmented correlation-consistent valence-triple- $\zeta$ basis set with polarization functions [20] (AVTZ) using the Molpro 2002.6 program suite [21]. The DFT calculations were carried out with the AVTZ basis set employing the PBE, BLYP, and TPSS exchange-correlation functionals [22-25] and using Gaussian 03 program [26]. Calculated interaction energies were corrected for a basis set superposition error (BSSE) using the counterpoise correction method [27].

All calculations were performed with fixed monomer geometries. The geometry of Gauss and Stanton $\left(\mathrm{r}_{\mathrm{CC}}=1.3915\right.$ $\AA, \mathrm{r}_{\mathrm{CH}}=1.0800 \AA$ ) was used for the benzene [28]. The geometries optimized at the $\operatorname{CCSD}(\mathrm{T}) / \mathrm{AVTZ}$ level were used for the ethylene $\left(\mathrm{r}_{\mathrm{CC}}=1.338 \AA, \mathrm{r}_{\mathrm{CH}}=1.084 \AA, \alpha=121.4 \mathrm{deg}\right)$ and for $\mathrm{H}_{2}\left(\mathrm{r}_{\mathrm{HH}}=0.742 \AA\right)$.

The $\mathrm{H}-\mathrm{H}$ and $\mathrm{C}-\mathrm{H}$ correction surfaces were constructed using 4900 (4-dimensional $\mathrm{R}, \alpha, \beta, \delta$ grid) points for $\mathrm{H}_{2} \ldots \mathrm{H}_{2}$ and 9800 (5-dimensional $\mathrm{R}, \alpha, \beta, \gamma, \delta$ grid) points for $\mathrm{C}_{2} \mathrm{H}_{4} \ldots \mathrm{H}_{2}$ calculated at the $\operatorname{CCSD}(\mathrm{T})$ and DFT (PBE, BLYP, TPSS) levels. For $\mathrm{C}_{2} \mathrm{H}_{4} \ldots \mathrm{C}_{2} \mathrm{H}_{4}$ only cuts along the $\mathrm{R}$ coordinate were calculated: total number of 448 structures for the Eth...Bz and $\mathrm{Bz} . . . \mathrm{Bz}$ complexes. One dimensional $R-\Omega_{S}$ correction curves were constructed using 66, 62, 52, 41 and 21 points (stepsize $0.1 \AA$ ) for $\mathrm{H}_{2} \ldots \mathrm{H}_{2}, \mathrm{C}_{2} \mathrm{H}_{4} \ldots \mathrm{H}_{2}$, $\mathrm{C}_{6} \mathrm{H}_{6} \ldots \mathrm{H}_{2}, \mathrm{C}_{2} \mathrm{H}_{4} \ldots \mathrm{C}_{2} \mathrm{H}_{4}$, and $\mathrm{C}_{6} \mathrm{H}_{6} \ldots \mathrm{C}_{2} \mathrm{H}_{4}$, respectively. 


\section{RESULTS}

\section{Ethylene...Benzene Complex}

The performance of the DFT/CCSD(T) correction scheme was tested for the Eth...Bz dimer. Potential energy curves were calculated for the " $\mathrm{S}$ " and " $\mathrm{T}$ " complexes (defined in Fig. (3)) at the $\operatorname{CCSD}(\mathrm{T}) / \mathrm{AVTZ}$ level, and these served as the reference data to compare with DFT/CCSD(T). Minimum energy distances obtained at the $\Omega$-DFTD and CCSD(T)/AVTZ levels are summarized in Table 1. Results obtained with the $\Omega^{(A)}$ and $\Omega_{S}^{(A)}$ models, are reported. It is apparent that all the exchange-correlation functionals used in $\Omega$-DFTD give the equilibrium distances within $0.02 \AA$ of the reference $\operatorname{CCSD}(\mathrm{T}) / \mathrm{AVTZ}$ geometry. Considering the fact that the potential energy curves are rather shallow, the agreement between $\operatorname{CCSD}(\mathrm{T})$ and $\Omega$-DFTD is very good. Equilibrium distances obtained with the one-dimensional $\Omega_{S}^{(A)}$-DFTD model are only slightly shorter than those obtained with anisotropic $\Omega^{(A)}$-DFTD model; apparently, neglecting the anisotropy of the DFT correction does not influence the minimum energy structures of the $\mathrm{S}$ and $\mathrm{T}$ complexes of Eth...Bz.

It should be noted that the potential energy curves obtained at the DFT level without any corrections are either purely repulsive (BLYP) or show a very small interaction energy (PBE and TPSS) and a minimum at relatively large distance (Fig. 3). Regardless the behavior of a particular exchange-correlation functional (whether it is purely repulsive or whether it gives some minimum on the potential energy curve), the $\Omega$-DFTD correction fixes the problem and final equilibrium distances are the same for all functionals investigated.

Table 1. Minimum Energy Distances $R$ (in Å) for the Eth...Bz Complex Calculated for the $S$ and $T$ Structures $^{\text {a }}$

\begin{tabular}{|c|c|c|c|c|}
\hline \multirow{2}{*}{ Method/Model } & \multicolumn{2}{|c|}{ S Structure } & \multicolumn{2}{c|}{ T Structure } \\
\cline { 2 - 5 } & $\Omega^{(A)}$ & $\Omega_{S}^{(A)}$ & $\Omega^{(A)}$ & $\Omega_{S}^{(A)}$ \\
\hline \hline PBE/CCSD(T) & 3.57 & 3.54 & 4.29 & 4.26 \\
\hline BLYP/CCSD(T) & 3.59 & 3.55 & 4.29 & 4.27 \\
\hline TPSS/CCSD(T) & 3.55 & 3.54 & 4.27 & 4.27 \\
\hline CCSD(T) & \multicolumn{3}{|c|}{3.57} & \multicolumn{2}{|c}{4.27} \\
\hline${ }^{a}$ see Fig. (3) for a definition.
\end{tabular}

Interaction energies of the Eth...Bz complex were calculated at the $\operatorname{CCSD}(\mathrm{T}) / \mathrm{AVTZ}$ level and with the $\Omega$-DFTD correction scheme along the potential energy curves of the $S$ and $\mathrm{T}$ structures (Fig. 3). The interaction energies calculated at the geometry of the minimum energy structure $\left(R_{\min }=\right.$ 3.57 and $4.27 \AA$ for the $\mathrm{S}$ and $\mathrm{T}$ structures, respectively, at the CCSD(T)/AVTZ level) are summarized in Table $\mathbf{2}$ together with the individual $\Delta E_{H H}, \Delta E_{C H}$, and $\Delta E_{C C}$ contributions to the correction term $\Delta E$.
The results obtained with the $\Omega^{(A)}$-DFTD model are discussed first. A consistent description of both the $\mathrm{S}$ and $\mathrm{T}$ structures along the potential energy curves is obtained for all the exchange-correlation functionals investigated. Around the minima at the potential energy curves the deviation from the reference $\operatorname{CCSD}(\mathrm{T}) / \mathrm{AVTZ}$ results does not exceed 0.1 $\mathrm{kcal} / \mathrm{mol}$ for any of the functionals considered. It is obvious that the error of the $\Omega$-DFTD model is even smaller for $R \geq$ $R_{\text {min }}$. This error is still rather small for $R_{E=0}<R<R_{\text {min }}$ ( $E_{\text {int }}=0$ at $R_{E=0}$ ); below $0.1 \mathrm{kcal} / \mathrm{mol}$ for PBE and BLYP and up to $0.25 \mathrm{kcal} / \mathrm{mol}$ for TPSS. Using the TPSS or PBE functionals, the $\Omega^{(A)}$-DFTD model systematically slightly overestimates and underestimates the Eth...Bz interaction, respectively. On the contrary, the BLYP functional leads to overestimated and underestimated interactions for the $\mathrm{S}$ and $\mathrm{T}$ structures, respectively. On average, $\Omega^{(A)}$-DFTD tends to overestimate the $\mathrm{S}$ structure by $0.05 \mathrm{kcal} / \mathrm{mol}$ and underestimate the $\mathrm{T}$ structure by $0.03 \mathrm{kcal} / \mathrm{mol}$. This results in a slightly lower energy separation of the $\mathrm{S}$ and $\mathrm{T}$ structures: $0.31,0.32$, and $0.26 \mathrm{kcal} / \mathrm{mol}$ for the PBE, TPSS, and BLYP functionals, in a reasonable agreement with the $\operatorname{CCSD}(\mathrm{T}) / \mathrm{AVTZ}$ results $(0.38 \mathrm{kcal} / \mathrm{mol})$.

The largest values of $\Delta E$ are found for the BLYP functional; that is about twice as large as for the PBE functional. The individual components of $\Delta E\left(\Delta E=\Delta E_{H H}+\Delta E_{C H}+\right.$ $\triangle E_{C C}$ ) obtained with various functionals are rather different. Using the PBE functional results in negligible $\Delta E_{H H}$, moderate $\Delta E_{C H}$, and a majority of $\Delta E$ correction comes from $\Delta E_{C C}$. Similarly for the TPSS functional the $\Delta E_{H H}$ correction term is negligible, however, the $\Delta E_{C H}$ and $\Delta E_{C C}$ corrections are of equal importance. The relative importance of the individual $\Delta E$ components is significantly different for the BLYP functional. A non-negligible negative $\Delta E_{H H}$ correction term indicates that the $\mathrm{H}-\mathrm{H}$ interaction is too repulsive at the BLYP level. This correction is due to the deficiency of BLYP to describe the interaction of the electron densities around two $\mathrm{H}$ atoms and not due to the lack of the dispersion component. When using the BLYP functional, $\Delta E_{C H}$ is greater than $\Delta E_{C C}$ for the equilibrium $\mathrm{S}$ and $\mathrm{T}$ geometries. In summary, the PBE and TPSS functionals behave similarly while the BLYP functional is rather different; in fact, this is obvious just from the uncorrected potential energy curves obtained for the individual functionals (Fig. 3).

In order to investigate the importance of the anisotropic part of the intermolecular energy correction $\Delta E$ the interaction between the benzene and ethylene was investigated with the $\Omega_{S}^{(A)}$-DFTD model. Going from the complete $\Omega^{(A)}$-DFTD model to the isotropic $\Omega_{S}^{(A)}$-DFTD model results in the increase of the Eth...Bz interaction energies. Relatively small change is observed for all functionals at the equilibrium geometry of the $\mathrm{S}$ complex. The difference between the $\Omega^{(A)}$ and $\Omega_{S}^{(A)}$-model description of the Eth...Bz complex becomes even smaller for larger intermolecular distances. For the description of the $\mathrm{T}$ structure at the minimum-energy distance the difference between the $\Omega^{(A)}$ - and $\Omega_{S}^{(A)}$-models is 

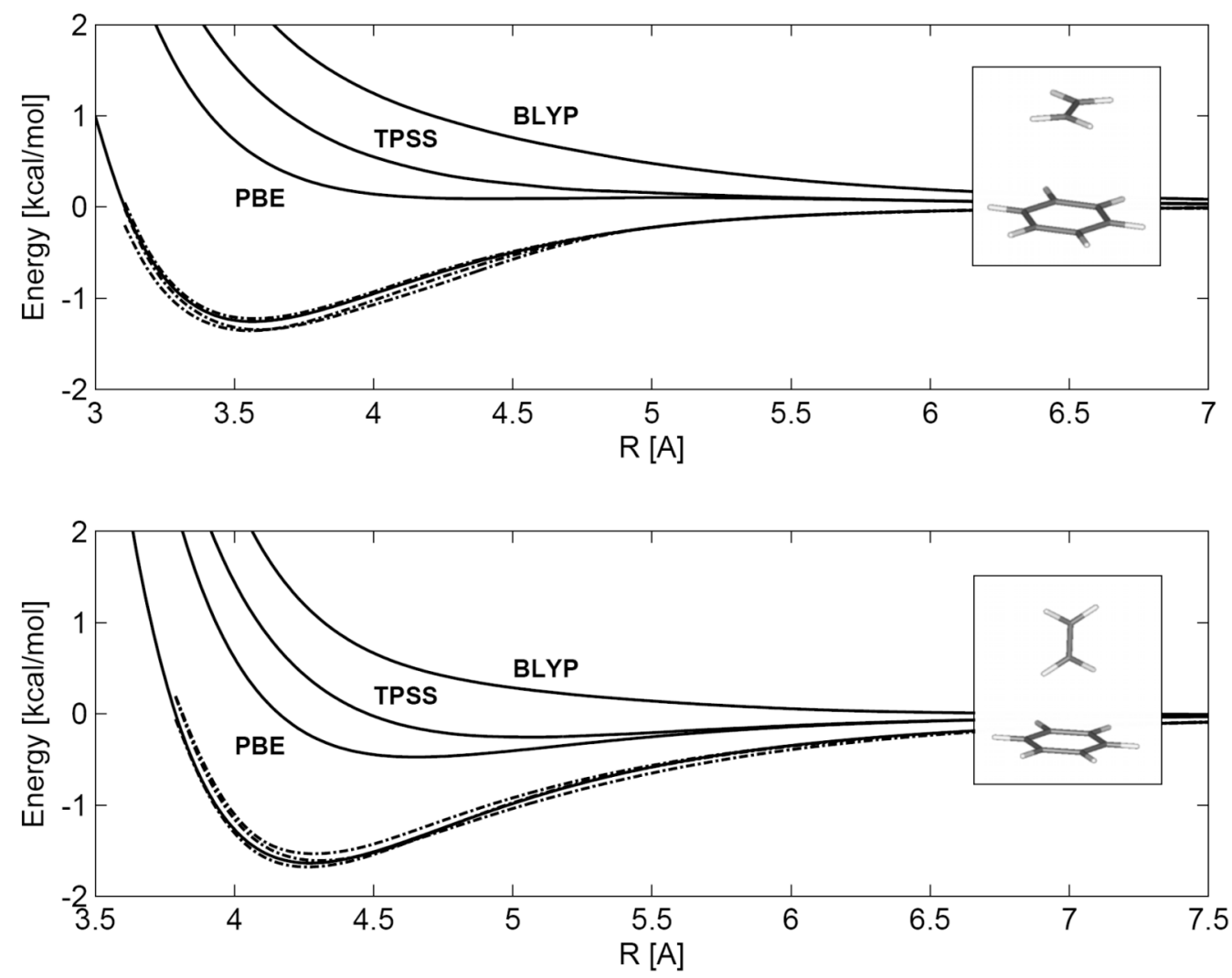

Fig. (3). Potential energy curves for the $S$ and $T$ structure of the Eth...Bz complex (upper and lower parts, respectively). The DFT (PBE, TPSS, and BLYP functionals employed) and $\operatorname{CCSD}(\mathrm{T})$ results depicted as solid lines; potential energy curves from the DFT/CCSD(T) correction scheme (the $\Omega^{(A)}$-DFTD model employed) depicted as dot-and-dash lines.

somewhat larger (up to $-0.16 \mathrm{kcal} / \mathrm{mol}$ ) than for the $\mathrm{S}$ structure. Still the effect of the anisotropy does not count for more than $10 \%$ of the interaction energy. Calculations of the interaction energies at shorter inter-molecular distances, $R_{E=0}<R$ $<R_{\text {min }}$ show that the difference between the $\Omega^{(A)}$ - and $\Omega_{S}^{(A)}$ models gradually increases for decreasing $R$, however, does not exceed $20 \%$ at this range.

The effect of the definition of the reference set was also investigated within the framework of the one-dimensional $\Omega_{S}$ model. An augmentation of the reference set by $\mathrm{H}_{2} \ldots \mathrm{Bz}$ and replacement of Eth...Eth by Eth...Bz (reference set B) led to additional improvements of the results (Table 2 ). The $\Omega_{S}^{(B)}$-DFTD model now gives exactly the same results as the CCSD(T)/AVTZ reference for the S structure; this is an obvious consequence of the reference set definition (reference set $\mathbf{B}$ now includes potential energy curve of the Eth...Bz complex in the $\mathrm{S}$ arrangement). However, even for the description of the T complex, the $\Omega_{S}^{(B)}$-DFTD model gives results of a comparable quality as the $\Omega^{(A)}$-DFTD model; with $\Omega_{S}^{(B)}$ giving a smaller spread for the individual DFT functionals.

\section{Benzene... Benzene Complex}

The geometry of the benzene dimer was optimized for the $\mathrm{S}$ and $\mathrm{T}$ structures using the $\Omega_{S}^{(A)}$-DFTD method and
BLYP functional. The geometries of monomers were fixed, and the $D_{6 h}$ and $C_{2 v}$ symmetry constraints were employed for the geometry optimization of the sandwich $(\mathrm{S})$ and T-shaped (T) structures, respectively. Optimized inter-molecular distances $R(R=3.96 \AA$ for $\mathrm{S}$ and $R=5.02 \AA$ for $\mathrm{T}$ structure $)$ are in a very good agreement with the data available from literature: 3.9 and $5.0 \AA$ for the $\mathrm{S}$ and $\mathrm{T}$ complexes, respectively, estimated with the $\operatorname{CCSD}(\mathrm{T})$ and large basis set (AVQZ and VQZ basis sets on $\mathrm{C}$ and $\mathrm{H}$ atoms, respectively) [30]. The same results were obtained at the SCSMP2/AVTZ* level (AVTZ* basis set consists of AVTZ and VTZ on C and H atoms, respectively) [29]. The geometry of the parallel-displaced (PD) structure optimized previously by Hill et al. [29] at the SCS-MP2/AVTZ* level was used $\left(R_{1}=\right.$ $3.6 \AA$ and $R_{2}=1.6 \AA$, for details see Fig. (1) in Ref. [29]). Single point energy calculations for the S, T, and PD structures were carried out using the $\Omega^{(A)}, \Omega_{S}^{(A)}$, and $\Omega_{S}^{(B)}$ correction schemes and the PBE, TPSS, and BLYP functionals. Results are reported in Table $\mathbf{3}$.

The results obtained with the $\Omega$-DFTD correction scheme should be compared with the CCSD(T)/AVTZ results. However, the largest coupled clusters calculations reported so far for the S, T, and PD complexes were performed with the modified AVTZ* basis set (defined above) [29]. For the highly symmetrical S structure Podeszwa et al. reported the CCSD(T)/AVTZ+b (AVTZ basis set on all atoms augmented with bond functions) interaction energy -1.583 $\mathrm{kcal} / \mathrm{mol}[31]$ that is very close to $\mathrm{CCSD}(\mathrm{T}) / \mathrm{AVTZ}^{*}$ value of 
Table 2. Interaction Energies of the Ethylene...Benzene Complex (S and T Structures) Calculated at the DFT, $\Omega-D F T$, and $\operatorname{CCSD}(\mathrm{T})$ Levels ${ }^{\mathrm{a}}, \Delta E$ Components Due to the H-H, H-C, and C-C Components are Reported. $\Omega$-DFT Interactions Calculated with the $\Omega^{(A)}, \Omega_{S}^{(A)}$, and $\Omega_{S}^{(B)}$ Models and the PBE, BLYP, and TPSS Functionals are Reported

\begin{tabular}{|c|c|c|c|c|c|c|c|}
\hline \multirow{2}{*}{ Model } & \multirow{2}{*}{ DFT Functional } & \multirow{2}{*}{$\frac{E_{\text {int }}}{\text { DFT }}$} & \multicolumn{4}{|c|}{ DFT/CCSD(T) Corrections } & \multirow{2}{*}{$\frac{E_{\text {int }}}{\mathrm{DFT} / \mathrm{CCSD}(\mathrm{T})}$} \\
\hline & & & $\Delta E_{H H}$ & $\Delta E_{C H}$ & $\Delta E_{C C}$ & $\Delta E$ & \\
\hline \multicolumn{8}{|c|}{ Structure $S(R=3.57 \AA): E(C C S D(T))=-1.26^{b}$} \\
\hline \multirow{3}{*}{$\Omega^{(A)}$} & PBE & 0.57 & -0.02 & -0.17 & -1.61 & -1.80 & -1.22 \\
\hline & BLYP & 2.24 & -0.40 & -1.76 & -1.43 & -3.59 & -1.35 \\
\hline & TPSS & 1.32 & 0.00 & -0.83 & -1.85 & -2.68 & -1.36 \\
\hline \multirow{3}{*}{$\boldsymbol{\Omega}_{S}^{(A)}$} & PBE & & 0.05 & -0.36 & -1.57 & -1.88 & -1.31 \\
\hline & BLYP & & -0.44 & -1.66 & -1.54 & -3.64 & -1.40 \\
\hline & TPSS & & 0.00 & -0.81 & -1.96 & -2.76 & -1.44 \\
\hline \multirow{3}{*}{$\Omega_{S}^{(B)}$} & PBE & & 0.05 & -0.51 & -1.37 & -1.83 & -1.26 \\
\hline & BLYP & & -0.44 & -1.57 & -1.49 & -3.50 & -1.26 \\
\hline & TPSS & & 0.00 & -0.86 & -1.72 & -2.58 & -1.26 \\
\hline \multicolumn{8}{|c|}{ Structure $T(R=4.27 A): \operatorname{E}(C \operatorname{CSD}(T))=-1.64^{b}$} \\
\hline \multirow{3}{*}{$\Omega^{(A)}$} & PBE & -0.23 & 0.01 & -0.37 & -0.94 & -1.30 & -1.53 \\
\hline & BLYP & 1.14 & -0.37 & -1.49 & -0.89 & -2.75 & -1.61 \\
\hline & TPSS & 0.38 & 0.01 & -1.04 & -1.02 & -2.06 & -1.68 \\
\hline \multirow{3}{*}{$\Omega_{S}^{(A)}$} & PBE & & 0.06 & -0.52 & -0.94 & -1.40 & -1.63 \\
\hline & BLYP & & -0.41 & -1.53 & -0.97 & -2.91 & -1.77 \\
\hline & TPSS & & 0.00 & -1.04 & -1.17 & -2.22 & -1.84 \\
\hline \multirow{3}{*}{$\Omega_{S}^{(B)}$} & PBE & & 0.06 & -0.65 & -0.82 & -1.42 & -1.65 \\
\hline & BLYP & & -0.41 & -1.50 & -0.92 & -2.84 & -1.70 \\
\hline & TPSS & & 0.00 & -1.07 & -1.03 & -2.10 & -1.72 \\
\hline
\end{tabular}

$-1.564 \mathrm{kcal} / \mathrm{mol}$. Therefore, it is likely that the CCSD(T)/AVTZ* values of Hill et al. [29] (used in Table 3 as reference values) are very closed to the not-yet-reported $\operatorname{CCSD}(\mathrm{T}) / \mathrm{AVTZ}$ values and we use the $\operatorname{CCSD}(\mathrm{T}) / \mathrm{AVTZ}$ * results as the reference data to compare with our $\Omega$-DFTD results obtained at the $\operatorname{CCSD}(\mathrm{T}) / \mathrm{AVTZ}$ reference level of theory.

Results obtained for the benzene dimer using the $\Omega^{(A)}$, $\Omega_{S}^{(A)}$, and $\Omega_{S}^{(B)}$ correction schemes are in the same relation as found for Eth...Bz, however, the differences between the individual correction schemes are somewhat larger in the case of the benzene dimer. It is apparent from Table 3 that the $\Omega_{s}^{(B)}$-DFTD results are better than the $\Omega^{(A)}$-DFTD results and far better than the $\Omega_{S}^{(A)}$-DFTD results. The $\Omega_{S}^{(B)} \rightarrow \Omega_{S}^{(A)}$ change leads to the systematic increase of the benzene dimer interaction energies, a magnitude of which depends on the particular functional; up to $0.27,0.25$, and $0.45 \mathrm{kcal} / \mathrm{mol}$ were found for the PBE, BLYP, and TPSS functionals, respectively. Based on the comparison of the results obtained within the $\Omega^{(A)}$ and $\Omega_{S}^{(A)}$ correction schemes it is suggested that the anisotropy of the $\mathrm{DFT} / \mathrm{CCSD}(\mathrm{T})$ correction is smaller for the PBE functional than that for the TPSS and BLYP functionals; the PBE functional may, thus, be more suitable for the DFT/CCSD(T) correction.

With the PBE functional and $\Omega_{s}^{(B)}$-DFTD correction scheme the interaction energies of the benzene dimer at the $\mathrm{S}, \mathrm{T}$, and PD structures are $-1.56,-2.42$, and $-2.46 \mathrm{kcal} / \mathrm{mol}$, respectively, in excellent agreement with the reference $\operatorname{CCSD}(\mathrm{T}) / \mathrm{AVTZ}^{*}$ results $(-1.56,-2.49,-2.51 \mathrm{kcal} / \mathrm{mol}$, respectively).

\section{DISCUSSION}

It is apparent from the results presented above that within the DFT/CCSD(T) correction scheme any of the tested functionals can be successfully corrected for the description of the weak intermolecular interactions. Compared to the standard DFTD approach, a major advantage of the proposed correction scheme is in its ability to analyze the validity of the individual approximations adopted in a particular model. 
Table 3. Interaction Energies of the Bz...Bz Complex (S, T, and PD Structures) Calculated at the DFT, $\Omega$-DFT, and CCSD(T) Levels ${ }^{\mathrm{a}}, \Delta E$ Components Due to the H-H, H-C, and C-C Components are Reported, $\Omega$-DFT Interactions Calculated with the $\Omega^{(A)}, \Omega_{S}^{(A)}$, and $\Omega_{S}^{(B)}$ Models and the PBE, BLYP, and TPSS Functionals are Reported

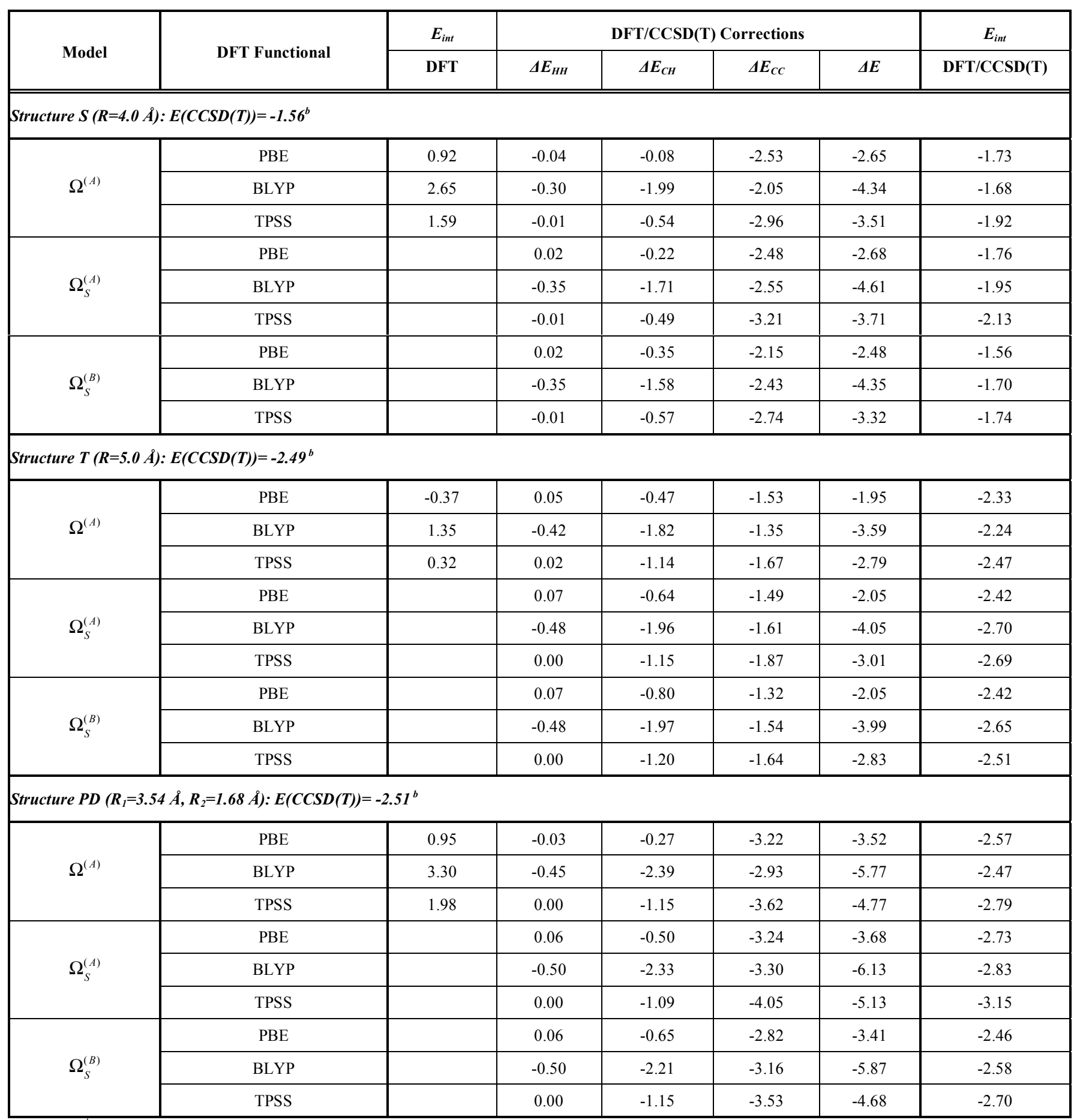

${ }^{a}$ In kcal/mol. ${ }^{b} \mathrm{CCSD}(\mathrm{T}) / \mathrm{AVTZ} *$ level, Ref. [29].

It is our goal to use the results presented in the previous section and analyze the suitability of a particular reference set, the importance of anisotropy, and the transferability of $\varepsilon_{X Y}^{\Omega}$ corrections.

The definition of the $\Omega$-DFTD method includes the specification of a reference set (e.g., $\Omega^{(A)}$ or $\Omega^{(B)}$ sets de- scribed above), the reference level of theory $(\operatorname{CCSD}(\mathrm{T}) /$ ATVZ used here), and the definition of the complexity of the $R-\Omega$ correction surfaces (either a fully anisotropic $\Omega$ correction surface or one-dimensional $\Omega_{S}$ correction curves were adopted by us). In general, a $\Omega \equiv \Omega^{(0)}$ model, where the reference set (0) includes just the investigated complexes, represents the exact $\Omega$-DFTD correction scheme within a 
DFT/CCSD(T) family (e.g., the $\Omega^{(B)}$ correction surface used for the Eth...Bz complex). A hierarchy of the $\Omega$-DFTD schemes is depicted in Fig. (4).

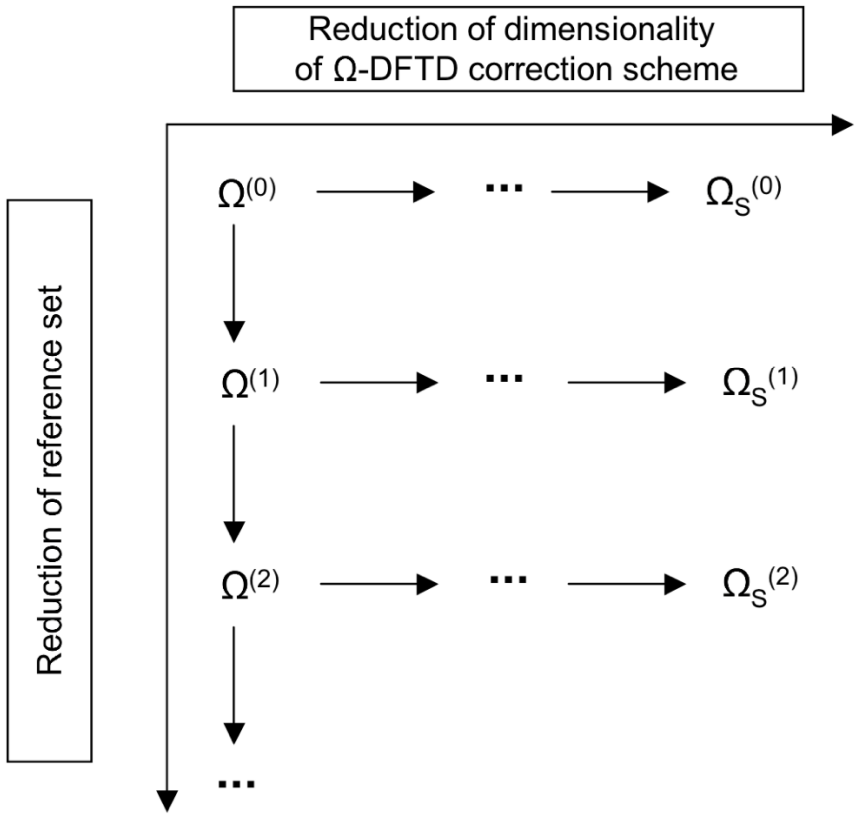

Fig. (4). Possible simplification of the $\Omega$-DFTD scheme. The horizontal direction represents the dimensionality reduction starting with the completely anisotropic $\Omega$ model and ending with the onedimensional $\Omega_{S}$ model for a given reference set. The vertical direction represents the reduction of reference sets.

For practical use, however, the $\Omega$-DFTD method requires a more approximate correction scheme than $\Omega^{(0)}$.

There are two possibilities how to reduce this complexity: a reduction of the correction-surface dimensionality and a use of a smaller reference set. First, the dimensionality of the complete $R-\Omega$ correction surface can be reduced (change along the horizontal direction in Fig. (4)); the largest reduction possible leads to a one-dimensional $\Omega$-specific correction curve (e.g., $R-\Omega_{s}$ defined in Fig. (2)). (Onedimensional $\Omega$-non-specific description is used in the standard DFTD techniques.) The validity of this simplifying approximation depends on the importance of the anisotropy of the $\mathrm{DFT} / \mathrm{CCSD}(\mathrm{T})$ correction functions $\varepsilon_{X Y}^{\Omega}$. Second, changes in the reference set definition (along the vertical direction in Fig. (4)) may also lead to a substantial simplification of the $\Omega$-DFTD procedure. Validity of this approximation depends on the transferability of the correction functions $\varepsilon_{X Y}^{\Omega}$. As it was mentioned already, the advantage of the $\Omega$-DFTD procedure is in its ability to analyze validity of a particular approximation simply by changing the definition of the reference set and by changing the correction-surface dimensionality.

Using the notation summarized in Fig. (4) it is obvious that $\Omega^{(0)} \equiv \Omega^{(B)}$ in the case of the Eth...Bz complex, while this does not hold for $\mathrm{Bz} \ldots \mathrm{Bz}$ (the benzene dimer is not a part of reference set $\mathbf{B}$ ). It should be pointed out that for the $\mathrm{S}$ structure of the Eth...Bz complex the $R-\Omega_{S}^{(B)}$ correction curve is a cut of the complete $R-\Omega^{(B)}$ correction surface along $R$. Therefore, the results obtained within $\Omega_{S}^{(B)}$-DFTD for the $S$ structure are exact (indeed, the $\Omega_{S}^{(B)}$ interaction energies reported for the $\mathrm{S}$ structure in Table $\mathbf{2}$ are the same as the values obtained at the reference level). In terms of Fig. (4) notation the results obtained with the following $\Omega$ models are available: $\Omega^{(0)}, \Omega^{(1)}, \Omega_{s}^{(0)}$, and $\Omega_{S}^{(1)}$ for Eth...Bz and $\Omega^{(1)}, \Omega^{(2)}, \Omega_{s}^{(1)}$, and $\Omega_{s}^{(2)}$ for the benzene dimer. Note that the $\Omega^{(0)}$ results are just the reference level values. The differences between these $\Omega$ models are analyzed below.

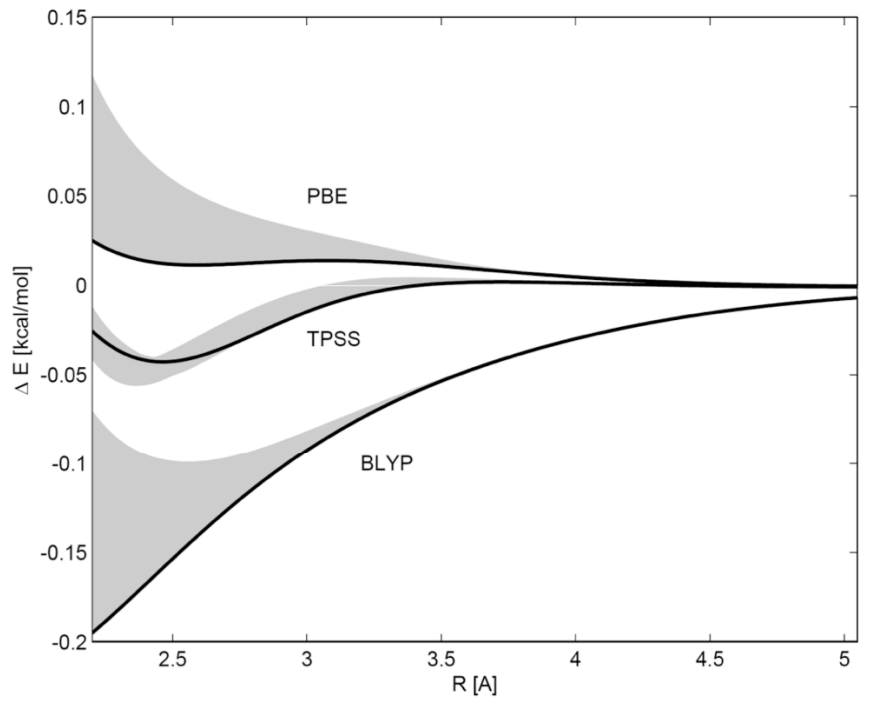

Fig. (5). DFT/CCSD(T) correction due to the $\mathrm{H}-\mathrm{H}$ interaction, $\Delta E_{H H}$, for the PBE, TPSS, and BLYP functionals as a function of the $\mathrm{H}-\mathrm{H}$ distance. One-dimensional correction curves $R-\Omega_{S}^{(A)}$ depicted as solid lines; complete $R-\Omega^{(A)}$ correction surfaces, projected to the inter-molecular distance $R$, depicted as grey-shaded areas.

Results reported in the previous section can be analyzed from the point of view of the transferability of the correction functions $\varepsilon_{X Y}^{\Omega}$. In the case of Eth...Bz complex the difference due to the reference sets $\mathbf{A}$ and $\mathbf{B}$ can be discussed for the complete $R-\Omega$ correction surface. The use of the reference set $\mathbf{A}$, that does not include benzene, leads to an unsystematic error of about $0.1 \mathrm{kcal} / \mathrm{mol}$. A similar difference due to the reference set definition can be observed when the onedimensional $R-\Omega_{S}$ correction curve is used. Significantly larger (but systematic) differences between the interaction energies obtained within the reference sets $\mathbf{A}$ and $\mathbf{B}$ are observed for the benzene dimer (only the one-dimensional $R-\Omega_{S}$ correction curves are available with both sets): up to $0.25 \mathrm{kcal} / \mathrm{mol}$ with the PBE and BLYP functionals and up to $0.45 \mathrm{kcal} / \mathrm{mol}$ with the TPSS functional. As for the transferability of correction functions $\varepsilon_{C C}^{\Omega}$ our results can be summarized as follows: (i) correction functions obtained from the ethylene dimer can be used for the Eth...Bz complex (error 
below $0.1 \mathrm{kcal} / \mathrm{mol}$ ), (ii) using $\varepsilon_{C C}^{\Omega}$ obtained from the ethylene dimer for the benzene dimer results in up to $0.5 \mathrm{kcal} / \mathrm{mol}$ error, and (iii) this error is reduced to $0.2 \mathrm{kcal} / \mathrm{mol}$ when $\varepsilon_{C C}^{\Omega}$ obtained from the Eth...Bz dimer are used.

The importance of the anisotropic part $\left(\Omega \rightarrow \Omega_{S}\right.$ transition) of the DFT/CCSD(T) correction is discussed next. From the data reported in Tables $\mathbf{2}$ and $\mathbf{3}$ it is apparent that the importance of the anisotropy of $\Delta E$ increases with the decreasing quality of the reference set. Reducing the dimensionality of the correction surface always leads to the increase of interaction energies: (i) $\Omega^{(0)} \equiv \Omega^{(B)} \rightarrow \Omega_{S}^{(B)}$ for Eth...Bz leads to only $0.01-0.08 \mathrm{kcal} / \mathrm{mol}$ change, (ii) $\Omega^{(1)} \equiv \Omega^{(A)} \rightarrow \Omega_{S}^{(A)}$ for Eth...Bz gives $0.05-0.16 \mathrm{kcal} / \mathrm{mol}$ change, and (iii) $\Omega^{(2)} \equiv \Omega^{(A)} \rightarrow \Omega_{S}^{(A)}$ for Bz...Bz gives 0.03$0.50 \mathrm{kcal} / \mathrm{mol}$ change. It should be noted that the PBE functional is least dependent on the dimensionality reduction (anisotropy).

To gain an additional insight on the importance of the anisotropic part due to the $\mathrm{H}-\mathrm{H}$ interactions, $\Delta E_{H H}$, evaluated for the individual functionals using the one-dimensional correction curves $R-\Omega_{S}$ and full $R-\Omega$ correction surfaces are depicted in Fig. (5). For the inter-molecular H-H distance larger than $3.5 \AA$ the $\Delta E_{H H}$ does not depends on the angular parameters $\Omega$. However, for shorter $\mathrm{H}-\mathrm{H}$ separations the anisotropic part of $\Delta E_{H H}$ starts to play a role; note that for the systems, where the inter-system $\mathrm{H}-\mathrm{H}$ distances are below 3.5 $\AA$, the use of the one-dimensional representation leads to a systematic overestimation of the interaction energies (except for TPSS where it can be over- or underestimated). Nevertheless, for the systems investigated here, the inter-molecular $\mathrm{H}-\mathrm{H}$ distances are always above $3.5 \AA$. A similar analysis can be performed for the $\Delta E_{C H}$ and $\Delta E_{C C}$ corrections, however, it cannot be easily visualized as for $\Delta E_{H H}$ (Fig. 5). The $\Delta E_{H H}, \Delta E_{C H}$, and $\Delta E_{C C}$ contributions to $\Delta E$ reported in Tables 2 and 3 for $\Omega^{(A)}-, \Omega_{S}^{(A)}$-, and $\Omega_{S}^{(B)}$-DFTD show that the $\Delta E_{C H}$ and $\triangle E_{C C}$ components depend on the dimensionality of the correction surface significantly more than the $\Delta E_{H H}$ component.

It is apparent that the errors due to approximations in the reference set and in the complexity of the correction surfaces correlate. This can be understood in terms of analysis of the electrostatic component of the interaction energy of the Eth...Bz and Bz...Bz complexes that is driven by the quadrupole-quadrupole interaction in both cases. The quadrupole moment $(\Theta)$ is underestimated at the DFT level $(-23.0,-24.1$, $-24.5 \times 10^{-40}$ C. $\mathrm{m}^{2}$ using the BLYP, TPSS, and PBE functionals, respectively) compared to the reference CCSD(T)/AVTZ level $\left(-26.1 \times 10^{-40}\right.$ C. $\left.\mathrm{m}^{2}\right)$. The electric quadrupole of the ethylene is also underestimated at the DFT level compared to $\operatorname{CCSD}(\mathrm{T})$, but to a smaller extent than in the benzene. Consequently, the $\Omega$-DFTD correction scheme implicitly includes some correction of the electrostatic $\Theta-\Theta$ interaction. $\varepsilon_{C C}^{\Omega}$ obtained from set $\mathbf{A}$ implicitly includes smaller correction of the $\Theta-\Theta$ interaction of the ethylene dimer; using these correction functions for the benzene dimer results in an insufficient correction of the $\Theta-\Theta$ interaction. On the contrary, the set $\mathbf{B}$ includes the Eth...Bz complex, thus, the $\varepsilon_{C C}^{\Omega}$ correction functions account better for the error in the $\Theta$ description of the benzene. This is clear from the results in Table 3. It follows that the anisotropy of the $\Delta E$ correction reported here is for a large part due to the DFT deficiency to describe the electric moments of monomers precisely. The $\Omega$-DFTD correction scheme can only fix the problems connected with the electrostatic component of the interaction when the monomer molecules are included in the reference set.

\section{CONCLUSIONS}

A novel DFT/CCSD $(\mathrm{T})$ correction scheme denoted as $\Omega$ DFTD was proposed for precise calculations of weakly interacting molecular systems. Main features distinguishing the $\Omega$-DFTD method from a general DFTD approach are: (i) A proper treatment of the anisotropy of the DFT/CCSD(T) corrections is achieved by introducing the $\Omega$ dependence into the DFTD equations, and (ii) The $\Omega$-DFTD calculation involves neither adjustable parameters $\left(\mathrm{C}_{6}\right.$ coefficients) nor artificial constraints on the functional form of the $\Omega$-DFTD correction (damping functions, combination rules). In this work the reliability and accuracy of the proposed method are clearly demonstrated on the ethylene-benzene and benzenebenzene complexes. Using the PBE exchange correlation functional and reference set $\mathbf{B}$, the $\Omega$-DFTD results for the benzene dimer are within $0.1 \mathrm{kcal} / \mathrm{mol}$ of the reference CCSD(T)/AVTZ level for all the considered structures, despite the fact that the benzene dimer is not included in the reference set.

Particular attention is paid to the analysis of assumptions commonly used in the DFTD calculations. We believe that the $\Omega$-DFTD method represents a valuable analytic tool that can be used for better understanding of the behavior and limits of the currently used empirically corrected DFT approaches. From this point of view the results of the present study can be summarized as follows:

The role of the anisotropy increases with simplifications in the reference set. The $\Omega$-DFTD anisotropy can be measured as an angular dependence of the DFT error with respect to the reference level of theory. Thus, we conclude that the mean $\Omega^{(n)}-\Omega_{s}^{(n)}$ energy difference increases with $n$ (vertical arrow in Fig. (4)). The anisotropy effect for the exact reference set $\left(\Omega^{(0)}-\Omega_{s}^{(0)}\right)$ seems to be negligible for all studied systems (Eth...Eth, Eth...Bz, Bz...Bz).

The error due to limited transferability of the DFT/CCSD $(T)$ correction measured as the $\Omega^{(n)}-\Omega^{(0)}$ energy difference is larger than the $\Omega^{(0)}-\Omega_{S}^{(0)}$ energy difference. This leads us to the conclusion that a suitable choice of the reference system is more important than a proper treatment of the $\Omega$-DFTD anisotropy.

In order to unambiguously clarify observed behavior of $\Omega$-DFTD we plan to investigate the DFT errors in more detail including errors originated in the DFT description of 
monomers. Obviously, some DFT errors may not be transferable, and only way around is a better choice of the reference system. Thus, we plan to employ the proposed computational methodology for the construction of the global PES of selected PAH clusters using the extended set of the $\Omega_{S}^{(n)}$ models.

\section{ACKNOWLEDGEMENTS}

This research is supported by the Grant Agency of the Academy of Sciences of the Czech Republic (grant No. IAA400550613). Work at the Institute of Organic Chemistry and Biochemistry (MR, PN, and $\mathrm{OB}$ ) is supported by the Ministry of Education, Youth and Sports of the Czech Republic (grant No. LC512 and research project No. Z4 055 0506). Work at the Charles University in Prague (PS) is supported by the Ministry of Education, Youth and Sports of the Czech Republic (research project No. 0021620835). The grant No. 203/06/0420 from the Grant Agency of the Czech Science Foundation is also acknowledged (O.B.).

\section{REFERENCES}

[1] Rapcewicz K, Ashcroft NW. Fluctuation attraction in condensed matter - a nonlocal functional-approach. Phys Rev B 1991; 44(8): 4032-5.

[2] Andersson Y, Langreth DC, Lundqvist BI. van der Waals interactions in density-functional theory. Phys Rev Lett 1996; 76(1): 1025 .

[3] Dobson JF, Dinte BP. Constraint satisfaction in local and gradient susceptibility approximations: Application to a van der Waals density functional. Phys Rev Lett 1996; 76(11): 1780-83.

[4] Wesolowski TA, Tran F. Gradient-free and gradient-dependent approximations in the total energy bifunctional for weakly overlapping electron densities. J Chem Phys 2003; 118(5): 2072-80.

[5] Kohn W, Meir Y, Makarov DE. vanderWaals energies in density functional theory. Phys Rev Lett 1998; 80(19): 4153-6.

[6] Misquitta, AJ, Jeziorski B, Szalewicz K. Dispersion energy from density-functional theory description of monomers. Phys Rev Lett 2003: 91(3).

[7] Misquitta AJ, Podeszwa R, Jeziorski B, Szalewicz K. Intermolecular potentials based on symmetry-adapted perturbation theory with dispersion energies from time-dependent density-functional calculations. J Chem Phys 2005; 123(21).

[8] Hesselmann A, Jansen G, Schutz M. Density-functional theorysymmetry-adapted intermolecular perturbation theory with density fitting: A new efficient method to study intermolecular interaction energies. J Chem Phys 2005; 122(1): 014103.

[9] Elstner M, Hobza P, Frauenheim T, Suhai S, Kaxiras E. Hydrogen bonding and stacking interactions of nucleic acid base pairs: A density-functional-theory based treatment. J Chem Phys 2001; 114(12): 5149-55
[10] Grimme S. Accurate description of van der Waals complexes by density functional theory including empirical corrections. J Computational Chem 2004; 25(12): 1463-73.

[11] Wu Q, Yang WT. Empirical correction to density functional theory for van der Waals interactions. J Chem Phys 2002; 116(2): 515-24.

[12] Zimmerli U, Parrinello M, Koumoutsakos P. Dispersion corrections to density functionals for water aromatic interactions. J Chem Phys 2004; 120(6): 2693-9.

[13] Grimme S. Semiempirical GGA-type density functional constructed with a long-range dispersion correction. J Comput Chem 2006; 27(15): 1787-99.

[14] Jurecka P, Cerny J, Hobza P, Salahub DR. Density functional theory augmented with an empirical dispersion term. Interaction energies and geometries of 80 noncovalent complexes compared with ab initio quantum mechanics calculations. J Comput Chem 2007; 28(2): 555-69.

[15] Ahlrichs R, Penco R, Scoles G. Chem Phys, 1977; 19: 119.

[16] Johnson ER, Becke AD. A post-Hartree-Fock model of intermolecular interactions. J Chem Phys 2005; 123(2): 024101.

[17] Ho TS, Rabitz H. A general method for constructing multidimensional molecular potential energy surfaces from ab initio calculations. J Chem Phys 1996; 104(7): 2584-97.

[18] Soldán P, Hutson JM. On the long-range and short-range behavior of potentials from reproducing kernel Hilbert space interpolation. J Chem Phys 2000; 112(9): 4415-6.

[19] Cizek J. The CCSD(T) method. Adv Chem Phys 1969; 14: 35.

[20] Dunning TH. Gaussian-Basis Sets for Use in Correlated Molecular Calculations.1. The atoms boron through neon and hydrogen. J Chem Phys 1989; 90(2): 1007-23.

[21] Molpro is a package of ab initio programs written by: Amos RD, Bernhardsson A, Berning P, et al.

[22] Becke AD. Density-functional exchange-energy approximation with correct asymptotic-behavior. Phys Rev A 1988; 38(6): 30983100 .

[23] Lee C, Yang W, Parr RG. LYP. Phys Rev B: Condens Matter 1988; 37: 785.

[24] Perdew JP, Burke K, Ernzerhof M. Generalized gradient approximation made simple. Phys Rev Lett 1996; 77(18): 3865-8.

[25] Tao JM, Perdew PJ, Staroverov NV, Sicuseria EG. Climbing the density functional ladder: Nonempirical meta-generalized gradient approximation designed for molecules and solids. Phys Rev Lett 2003; 91(14): 146401.

[26] Frisch MJ, Trucks GW, Schlegel HB, et al. Gaussian 03. 2003; Gaussian Inc.: Pittsburgh PA.

[27] Boys SF, Bernardi F. Mol Phys 1970; 19: 553

[28] Gauss J, Stanton JF. The equilibrium structure of benzene. J Phys Chem A, 2000; 104(13): 2865-8.

[29] Hill JG, Platts JA, Werner HJ. Calculation of intermolecular interactions in the benzene dimer using coupled-cluster and local electron correlation methods. Phys Chem Chem Phys 2006; 8(35): 4072-4078

[30] Sinnokrot MO, Sherrill CD. Highly accurate coupled cluster potential energy curves for the benzene dimer: Sandwich, T-shaped, and parallel-displaced configurations. J Phys Chem A 2004; 108(46): 10200-10207.

[31] Podeszwa R, Bukowski R, Szalewicz K. Potential energy surface for the benzene dimer and perturbational analysis of pi-pi interactions. J Phys Chem A 2006; 110(34): 10345-54. 